\title{
On sortable intervals of monomials
}

\author{
V. Bonanzinga and S. Eliahou
}

\begin{abstract}
In 1996, in his study of Gröbner bases of toric ideals, Sturmfels introduced a sorting operator on pairs of monomials of degree $d$ in $n$ variables. This gave rise to the notion of sortable sets, namely sets $B$ of monomials of degree $d$ such that $B \times B$ is preserved by that operator. In this paper, we determine all lex-intervals or revlex-intervals of monomials which are sortable. The solution involves the notion of greatest common prefix.
\end{abstract}

\section{Introduction}

Throughout this paper, we shall let $S=K\left[x_{1}, \ldots, x_{n}\right]$ denote the polynomial ring in the variables $x_{1}, \ldots, x_{n}$ over a field $K$. We view $S$ as a graded algebra endowed with the standard grading given by $\operatorname{deg}\left(x_{i}\right)=1$ for all $i$. We denote by $S_{n, d}$ the set of monomials $x_{1}^{a_{1}} \cdots x_{n}^{a_{n}}$ in $S$ of degree $a_{1}+\cdots+a_{n}=d$.

In his study of Gröbner bases of toric ideals [5], Sturmfels introduced the following sorting operator on pairs of monomials in $S_{n, d}$.

Definition 1.1. The operator

$$
\begin{array}{ccc}
\text { sort: } \quad S_{n, d} \times S_{n, d} & \longrightarrow & S_{n, d} \times S_{n, d} \\
(u, v) & \longmapsto & \left(u^{\prime}, v^{\prime}\right)
\end{array}
$$

is defined as follows. For any $u, v \in S_{n, d}$, write $u v=x_{k_{1}} x_{k_{2}} \ldots x_{k_{2 d}}$ with non-decreasing indices $1 \leq k_{1} \leq k_{2} \leq \cdots \leq k_{2 d} \leq n$. We then set

$$
\begin{aligned}
& u^{\prime}=x_{k_{1}} x_{k_{3}} \ldots x_{k_{2 d-1}}, \\
& v^{\prime}=x_{k_{2}} x_{k_{4}} \ldots x_{k_{2 d}} .
\end{aligned}
$$

Key Words: Sortable sets, Lex-intervals.

2010 Mathematics Subject Classification: Primary 05E40

Received: 16.02 .2015

Accepted: 02.03.2015 
Observe that $u^{\prime}, v^{\prime}$ still belong to $S_{n, d}$ and that $\operatorname{sort}(u, v)=\operatorname{sort}(v, u)$.

Subsets $B \subset S_{n, d}$ which behave well with respect to this operator, i.e. such that

$$
\operatorname{sort}(B \times B) \subset B \times B,
$$

are said to be sortable. These sets are of special interest, in particular because they give rise to toric ideals which have quadratic Gröbner bases and to $K$ algebras which are Koszul. See $[1,2,3,4,5]$ for related information. It is difficult in general to describe families of sortable sets. Here, we shall focus on those subsets of $S_{n, d}$ which constitute an interval under the lexicographic or the reverse lexicographic order on $S_{n, d}$. Among them, we shall determine precisely those which are sortable.

Recall the definition of the lexicographic order on $S_{n, d}$. Given $\mathbf{a}=\left(a_{1}, \ldots, a_{n}\right), \mathbf{b}=\left(b_{1}, \ldots, b_{n}\right) \in \mathbb{N}^{n}$ such that $\sum_{i} a_{i}=\sum_{i} b_{i}=d$, we write

$$
x_{1}^{a_{1}} \cdots x_{n}^{a_{n}}>_{\text {lex }} x_{1}^{b_{1}} \cdots x_{n}^{b_{n}}
$$

if and only if the leftmost nonzero coordinate of $\mathbf{a}-\mathbf{b}$ is positive. Equivalently, let

$$
u=x_{i_{1}} \cdots x_{i_{d}}, v=x_{j_{1}} \cdots x_{j_{d}} \in S_{n, d}
$$

with $i_{1} \leq \cdots \leq i_{d}, j_{1} \leq \cdots \leq j_{d}$. Then $u>_{\text {lex }} v$ if and only if the leftmost nonzero coordinate of $\left(i_{1}-j_{1}, \ldots, i_{d}-j_{d}\right)$ is negative. Actually, we shall mostly omit the subscript and simply write $\geq$ instead of $\geq_{\text {lex }}$.

We now recall the notions of lex-segments and lex-intervals.

Definition 1.2. For $v \in S_{n, d}$, the lex-segment $L(v)$ is the set of all monomials $u \in S_{n, d}$ satisfying $u \geq v$. More generally, for $v_{1} \geq v_{2} \in S_{n, d}$, the lex-interval $L\left(v_{1}, v_{2}\right)$ is the set of all monomials $u \in S_{n, d}$ satisfying $v_{1} \geq u \geq v_{2}$.

Here is a short description of the content of this paper. In Section 2, we establish properties of the sort operator which will help us compare the monomial pair $(u, v)$ with $\operatorname{sort}(u, v)$. In Section 3, we define sets $B_{\mathrm{iw}}(v)$, consisting of all monomials $u \in S_{n, d}$ which are index-wise smaller than or equal to a given $v \in S_{n, d}$, and we show that these sets are always sortable. In Section 4 , we show that for $v \in S_{n, d}$, the lex-segment $L(v)$ is sortable if and only if $L(v)$ coincides with $B_{\mathrm{iw}}(v)$. This necessary and sufficient condition is then turned into a simple criterion in Section 5 , thereby answering the problem of characterizing sortable lex-segments originally formulated in [2]. In order to go further and study the sortability of lex-intervals, we shall need the notion of greatest common prefix discussed in Section 6. This allows us to determine all sortable lex-intervals in Section 7. Finally, in Section 8, we obtain analogous sortability criteria for revlex-intervals. 


\section{Some properties of the sort operator}

We first introduce some terminology. Given a monomial $u \in S_{n, d}$, written as $u=x_{i_{1}} \cdots x_{i_{d}}$ with $1 \leq i_{1} \leq \cdots \leq i_{d} \leq n$, we shall usually denote its index multiset by the corresponding capital letter, that is

$$
U=\left[i_{1}, \ldots, i_{d}\right]
$$

Actually, we shall rather write

$$
U=\left[i_{1} \leq \cdots \leq i_{d}\right],
$$

since knowing the ordering of the elements of $U$ is useful in the present context. Note that for $u, v \in S_{n, d}$, with index multisets $U, V$, the index multiset of their product $u v \in S_{n, 2 d}$ is given by the multiset sum*

$$
U \uplus V \text {. }
$$

We now establish a few properties of the sort operator. For any $u, v \in S_{n, d}$, we shall keep the notation

$$
\left(u^{\prime}, v^{\prime}\right)=\operatorname{sort}(u, v)
$$

throughout the rest of this section.

Lemma 2.1. For all $u, v \in S_{n, d}$, we have $u v=u^{\prime} v^{\prime}$.

Proof. This is immediate, since the index multiset of $u^{\prime} v^{\prime}$ is, by construction, the same as that of $u v$.

Lemma 2.2. For all $u, v \in S_{n, d}$ such that $u \geq v$, there are only two possibilities:

$$
\begin{array}{r}
\text { either } u \geq u^{\prime} \geq v^{\prime} \geq v, \\
\text { or } u^{\prime} \geq u \geq v \geq v^{\prime} .
\end{array}
$$

Proof. Assume for a contradiction that $u \geq u^{\prime}$ and $v>v^{\prime}$. Then we get $u v>u^{\prime} v^{\prime}$, in contradiction with Lemma 2.1. The proof for the other case is similar.

In order to determine which of these alternatives the pair $(u, v)$ satisfies, we introduce a key index $r=\delta(u, v)$ and three types of monomial pairs.

\footnotetext{
*For finite multisets $U=\left[i_{1}, \ldots, i_{p}\right], V=\left[j_{1}, \ldots, j_{q}\right]$, their multiset sum is defined as $U \uplus V=\left[i_{1}, \ldots, i_{p}, j_{1}, \ldots, j_{q}\right]$.
} 
Definition 2.3. Let $u, v \in S_{n, d}$ such that $u \geq v$. Denote $u=x_{i_{1}} \cdots x_{i_{d}}$, $v=x_{j_{1}} \cdots x_{j_{d}}$ with $i_{1} \leq \cdots \leq i_{d}$ and $j_{1} \leq \cdots \leq j_{d}$, and set $i_{0}=j_{0}=0$. We shall denote by $\delta(u, v)$ the maximal integer $r$ such that $0 \leq r \leq d$ and

$$
i_{0} \leq j_{0} \leq i_{1} \leq j_{1} \leq \ldots \leq i_{r} \leq j_{r} .
$$

Still with $r=\delta(u, v)$, we say that the pair $(u, v)$ is of

- type 0 if $r=d$,

- type 1 if $r<d$ and $j_{r} \leq i_{r+1} \leq i_{r+2}<j_{r+1}$,

- type 2 if $r<d$ and $j_{r} \leq j_{r+1}<i_{r+1}$.

Observe that, by construction, every pair of monomials $(u, v)$ with $u \geq v$ is of one of the above three types, and uniquely so.

Proposition 2.4. Let $u, v \in S_{n, d}$ with $u \geq v$. Then the pair $(u, v)$ is of

- type 0 if and only if $\left(u^{\prime}, v^{\prime}\right)=(u, v)$;

- type 1 if and only if $u>u^{\prime}>v^{\prime}>v$;

- type 2 if and only if $u^{\prime}>u>v>v^{\prime}$.

Proof. Denote $u=x_{i_{1}} \cdots x_{i_{d}}, v=x_{j_{1}} \cdots x_{j_{d}}$ with $i_{1} \leq i_{2} \leq \cdots \leq j_{d}$ and $j_{1} \leq j_{2} \leq \cdots \leq j_{d}$. If $(u, v)$ is of type 0 , i.e. if $r=d$, we have

$$
i_{1} \leq j_{1} \leq \ldots \leq i_{d} \leq j_{d}
$$

whence $u^{\prime}=u$ and $v^{\prime}=v$ by definition of the sorting operator. Assume now $r<d$. We examine in turn the cases where $(u, v)$ is of type 1 and of type 2 . We shall denote by $U^{\prime}, V^{\prime}$ the index multiset of $u^{\prime}, v^{\prime}$, respectively.

Type 1. We have $j_{r} \leq i_{r+1} \leq i_{r+2}<j_{r+1}$. Thus, in the process of constructing $\left(U^{\prime}, V^{\prime}\right)$, we get

$$
\left(\begin{array}{l}
U^{\prime} \\
V^{\prime}
\end{array}\right)=\left(\begin{array}{lllll}
i_{1} & \cdots & i_{r} & i_{r+1} & \cdots \\
j_{1} & \cdots & j_{r} & i_{r+2} &
\end{array}\right)
$$

Since $i_{r+2}<j_{r+1}$, we have $v^{\prime}>v$, and hence $u>u^{\prime}$ by Lemma 2.2, as stated. Incidentally, we must have $r \leq d-2$ in this case.

Type 2. We have $j_{r} \leq j_{r+1}<i_{r+1}$. Then here, in the process of constructing $\left(U^{\prime}, V^{\prime}\right)$, we get

$$
\left(\begin{array}{l}
U^{\prime} \\
V^{\prime}
\end{array}\right)=\left(\begin{array}{lllll}
i_{1} & \cdots & i_{r} & j_{r+1} & \cdots \\
j_{1} & \cdots & j_{r} & &
\end{array}\right)
$$


Since $j_{r+1}<i_{r+1}$, we have $u^{\prime}>u$, whence $v>v^{\prime}$ by Lemma 2.2. Incidentally, we cannot have $r=0$ in this case, since $i_{1} \leq j_{1}$ by the assumption $u \geq v$.

Finally, since types 0,1 and 2 cover all possibilities, the implications proven so far are in fact equivalences.

\section{Sortable sets}

Definition 3.1. Let $B \subset S_{n, d}$ be a set of monomials of degree $d$ in $x_{1}, \ldots, x_{n}$. We say that $B$ is sortable if $B \times B$ is stable under the sort operator, i.e. if

$$
\operatorname{sort}(B \times B) \subset B \times B \text {. }
$$

Here is a known class of sortable sets. Given $\mathbf{a}=\left(a_{1}, \ldots, a_{n}\right) \in \mathbb{N}^{n}$, let

$$
S_{n, d}^{\mathbf{a}}=\left\{x_{1}^{c_{1}} \cdots x_{n}^{c_{n}} \in S_{n, d} \mid c_{i} \leq a_{i} \text { for all } i\right\} .
$$

Then $S_{n, d}^{\mathbf{a}}$ is sortable for all $\mathbf{a}=\left(a_{1}, \ldots, a_{n}\right) \in \mathbb{N}^{n}$. (See Proposition 6.11 in [2, p. 105].)

We now construct a new class of sortable sets. To this end, we introduce a partial order between monomials in $S_{n, d}$, denoted $\leq_{\text {iw }}$ and referred to as the index-wise partial order.

Definition 3.2. Let $u=x_{i_{1}} \cdots x_{i_{d}}, v=x_{j_{1}} \cdots x_{j_{d}}$ be monomials of degree $d$ with $i_{1} \leq i_{2} \leq \cdots \leq i_{d}$ and $j_{1} \leq j_{2} \leq \cdots \leq j_{d}$. We shall write

$$
u \leq_{\text {iw }} v
$$

if and only if $i_{\alpha} \leq j_{\alpha}$ for all $\alpha=1, \ldots, d$.

Definition 3.3. Let $v \in S_{n, d}$ be a monomial in $x_{1}, \ldots, x_{n}$ of degree d. Define

$$
B_{\mathrm{iw}}(v)=\left\{u \in S_{n, d} \mid u \leq_{\mathrm{iw}} v\right\} .
$$

For instance, for any $v \in S_{n, d}$, it is plain that $B_{\mathrm{iw}}(v)$ contains $x_{1}^{d}$. Note that if $u, v \in S_{n, d}$ and $u \leq_{\text {iw }} v$, then $u \geq v$ under the lexicographical order. This amounts to the set inclusion

$$
B_{\mathrm{iw}}(v) \subset L(v) .
$$

(See Lemma 4.1 below).

Observe that $B_{\mathrm{iw}}(v)$ is nothing else than the set of minimal generators of the principal Borel ideal $\langle v\rangle$ generated by $v$, see e.g. [6].

We now show that the sets $B_{\text {iw }}(v)$ constitute yet another class of sortable sets in $S_{n, d}$. 
Proposition 3.4. Let $v \in S_{n, d}$. Then $B_{\mathrm{iw}}(v)$ is sortable.

Proof. Let the index multiset of $v$ be $V=\left[j_{1} \leq \cdots \leq j_{d}\right]$. Let $u_{1}, u_{2} \in B_{\text {iw }}(v)$, with index multisets

$$
U_{1}=\left[h_{1} \leq \cdots \leq h_{d}\right], \quad U_{2}=\left[i_{1} \leq \cdots \leq i_{d}\right],
$$

respectively. By hypothesis, we have

$$
h_{\alpha}, i_{\alpha} \leq j_{\alpha}
$$

for all $\alpha=1, \ldots, d$. Let the index multiset of $u_{1} u_{2}=u_{1}^{\prime} u_{2}^{\prime}$ be

$$
W=\left[k_{1} \leq \cdots \leq k_{2 d}\right],
$$

where $W=U_{1} \uplus U_{2}$ is the multiset sum of $U_{1}, U_{2}$. By definition of the sorting operator, the index multisets of $u_{1}^{\prime}, u_{2}^{\prime}$ are

$$
\left[k_{1} \leq k_{3} \leq \cdots \leq k_{2 d-1}\right], \quad\left[k_{2} \leq k_{4} \leq \cdots \leq k_{2 d}\right],
$$

respectively. We must show that $u_{1}^{\prime}, u_{2}^{\prime} \leq_{i w} v$. Since $k_{2 \beta-1} \leq k_{2 \beta}$ for all $\beta$, this will follow at once from the following claim.

Claim. For all $\beta=1, \ldots, d$, we have

$$
k_{2 \beta} \leq j_{\beta} .
$$

Indeed, it follows from (1) that

$$
h_{\alpha}, i_{\alpha} \leq j_{\alpha} \leq j_{\beta}
$$

for all $1 \leq \alpha \leq \beta$. Thus, the multiset $W=U_{1} \uplus U_{2}$ contains at least $2 \beta$ elements which are bounded above by $j_{\beta}$, namely $\left[h_{1}, i_{1}, \ldots, h_{\beta}, i_{\beta}\right]$. But since $k_{1}, k_{2}, \ldots, k_{2 \beta}$ are the $2 \beta$ smallest elements in $W=U_{1} \uplus U_{2}$, it follows that

$$
k_{1}, \ldots, k_{2 \beta} \leq j_{\beta} .
$$

This proves the claim which, in turn, implies $u_{1}^{\prime}, u_{2}^{\prime} \in B_{\mathrm{iw}}(v)$.

Note that the sets $B_{\mathrm{iw}}(v)$ need not coincide with the sets $S_{n, d}^{\mathbf{a}}$. Indeed, let $v=x_{1} x_{3} \in S_{3,2}$. Then $B_{\text {iw }}(v)=\left\{x_{1}^{2}, x_{1} x_{2}, x_{1} x_{3}\right\}$. Thus, if we had $B_{\text {iw }}(v)=S_{3,2}^{\mathbf{a}}$ for some $\mathbf{a}=\left(a_{1}, a_{2}, a_{3}\right) \in \mathbb{N}^{3}$, we would then necessarily have $\mathbf{a}=(2,1,1)$. But now, observe that

$$
x_{2} x_{3} \in S_{3,2}^{(2,1,1)} \backslash B_{\mathrm{iw}}\left(x_{1} x_{3}\right) .
$$

Hence $B_{\text {iw }}\left(x_{1} x_{3}\right) \neq S_{3,2}^{\mathbf{a}}$ for all $\mathbf{a} \in \mathbb{N}^{3}$, as claimed. 


\section{When is a lex-segment sortable?}

In this section, we shall characterize all those lex-segments $L(v)$ which are sortable. We start by formalizing as a lemma an earlier observation comparing $B_{\text {iw }}(v)$ and $L(v)$.

Lemma 4.1. Let $v \in S_{n, d}$ be a monomial in $x_{1}, \ldots, x_{n}$ of degree $d$. Then $B_{\text {iw }}(v) \subset L(v)$.

Proof. Let $V=\left[j_{1} \leq \cdots \leq j_{d}\right]$ be the index multiset of $v$. Let $u \in S_{n, d}$ such that $u \leq_{\text {iw }} v$, with index multiset $U=\left[i_{1} \leq \cdots \leq i_{d}\right]$. Then we have

$$
i_{\alpha} \leq j_{\alpha}
$$

for all $\alpha=1, \ldots, d$. It follows that $u \geq v$, as desired.

Example 4.2. Let $v=x_{2}^{3} \in S_{3,3}$. Then we have

$$
\begin{aligned}
B_{\mathrm{iw}}(v) & =\left\{x_{1}^{3}, x_{1}^{2} x_{2}, x_{1} x_{2}^{2}, x_{2}^{3}\right\}, \\
L(v) & =\left\{x_{1}^{3}, x_{1}^{2} x_{2}, x_{1}^{2} x_{3}, x_{1} x_{2}^{2}, x_{1} x_{2} x_{3}, x_{1} x_{3}^{2}, x_{2}^{3}\right\} .
\end{aligned}
$$

We are now ready to state and prove our promised characterization.

Theorem 4.3. Let $v \in S_{n, d}$. Then $L(v)$ is sortable if and only if $L(v)=$ $B_{\text {iw }}(v)$.

Proof.

- If $L(v)=B_{\mathrm{iw}}(v)$, then $L(v)$ is sortable by Proposition 3.4.

- Conversely, assume that $L(v) \neq B_{\text {iw }}(v)$. Since $B_{\text {iw }}(v) \subset L(v)$ by Lemma 4.1, there exists some $u \in L(v) \backslash B_{\text {iw }}(v)$, i.e. such that $u>v$ and $u \mathbb{Z}_{\text {iw }} v$. Denote $u=x_{i_{1}} \cdots x_{i_{d}}$ with $i_{1} \leq i_{2} \leq \cdots \leq i_{d}$, and $v=x_{j_{1}} \cdots x_{j_{d}}$ with $j_{1} \leq j_{2} \leq \cdots \leq j_{d}$. Since $u>v$, there is an index $s \geq 1$ such that

$$
\begin{aligned}
& i_{\alpha}=j_{\alpha} \\
& i_{s}<j_{s} .
\end{aligned} \quad \text { for all } 1 \leq \alpha \leq s-1,
$$

Moreover, since $u \mathbb{Z}_{\mathrm{iw}} v$, there is an index $t \geq 1$ such that

$$
\begin{aligned}
& i_{\beta} \leq j_{\beta} \\
& i_{t}>j_{t} .
\end{aligned} \text { for all } 1 \leq \beta \leq t-1,
$$

It follows from the definition of $s, t$ that $s<t$. Since we do not know how $j_{\beta}$ compares with $i_{\beta+1}$ for $s+1 \leq \beta \leq t-1$, we have no precise control on $\delta(u, v)$ and we cannot determine the type of $(u, v)$. 
To get around this problem, we shall construct a new monomial $\bar{u} \in L(v) \backslash$ $B_{\text {iw }}(v)$, by suitably increasing all indices $i_{\beta}$ with $s+1 \leq \beta \leq t-1$, and for which the type of $(\bar{u}, v)$ will be easier to determine ${ }^{\dagger}$. So, let us define

$$
\bar{i}_{\beta}= \begin{cases}j_{\beta} & \text { if } s+1 \leq \beta \leq t-1 \\ i_{\beta} & \text { otherwise }\end{cases}
$$

and let $\bar{u}=x_{\bar{i}_{1}} \cdots x_{\bar{i}_{d}}$. We first claim that

$$
\bar{i}_{1} \leq \bar{i}_{2} \leq \cdots \leq \bar{i}_{d}
$$

Indeed, the inequality $\bar{i}_{\alpha} \leq \bar{i}_{\alpha+1}$ clearly holds for all $\alpha \in[1, d-1] \backslash\{s, t-1\}$, since in those cases it reduces to either $i_{\alpha} \leq i_{\alpha+1}$ or $j_{\alpha} \leq j_{\alpha+1}$. Now, for $\alpha=s$, we have

$$
\bar{i}_{s}=i_{s}<j_{s} \leq j_{s+1}=\bar{i}_{s+1},
$$

and for $\alpha=t-1$, we have

$$
\bar{i}_{t-1}=j_{t-1} \leq j_{t}<i_{t}=\bar{i}_{t} .
$$

This proves (4). Next, we claim that $\delta(\bar{u}, v)=t-1$. Indeed, relations (2) and (3) become

$$
\begin{aligned}
& \bar{i}_{\alpha}=j_{\alpha} \\
& \bar{i}_{s}<j_{s},
\end{aligned} \text { for all } 1 \leq \alpha \leq s-1,
$$

and

$$
\begin{aligned}
& \bar{i}_{\beta}=j_{\beta} \\
& \bar{i}_{t}>j_{t},
\end{aligned} \quad \text { for all } s+1 \leq \beta \leq t-1,
$$

respectively. As a first consequence, observe that $u \geq \bar{u}>v$. Moreover, by (5), (6) and the fact that the $j_{\alpha}$ are nondecreasing, we have

$$
\bar{i}_{1}=j_{1} \leq \bar{i}_{2}=j_{2} \leq \cdots \leq \bar{i}_{t-1}=j_{t-1} \leq j_{t}<\bar{i}_{t} .
$$

Thus, we have $\delta(\bar{u}, v)=t-1$ by definition of this index, and the pair $(\bar{u}, v)$ is of type 2 since $\bar{i}_{t-1} \leq j_{t-1} \leq j_{t}<\bar{i}_{t}$. It follows from Proposition 2.4 that $\bar{u}^{\prime}>\bar{u}>v>v^{\prime}$. Hence $L(v)$ is not sortable, as claimed.

\footnotetext{
${ }^{\dagger}$ If $s+1>t-1$, i.e. if $s=t-1$ since $s<t$, we simply end up with $\bar{u}=u$.
} 


\section{A simple criterion}

We shall now derive from Theorem 4.3 a simple criterion for recognizing when a given monomial $v \in S_{n, d}$ has the property that $L(v)$ is sortable or not.

Definition 5.1. Let $w$ be a monomial in the variables $x_{1}, \ldots, x_{n}$. If $w \neq 1$, we denote by $\min (w), \max (w)$ the smallest, respectively the largest, index of the variables dividing $w$. For $w=1$, we set $\min (1)=\infty$ and $\max (1)=0$.

For instance, if $w=x_{2}^{5} x_{3}^{2} x_{4}$, then $\min (w)=2$ and $\max (w)=4$.

Theorem 5.2. Let $v$ be a monomial in the variables $x_{1}, \ldots, x_{n}$. Then $L(v)$ is not sortable if and only if $v$ has a factor $w$ of degree 2 in $x_{2}, \ldots, x_{n-1}$.

Proof. Denote $v=x_{j_{1}} \ldots x_{j_{d}}$ with $j_{1} \leq \cdots \leq j_{d}$, and assume first that $L(v)$ is not sortable. By Theorem 4.3, there exists a monomial $u \in L(v) \backslash B_{i w}(v)$, i.e. satisfying $u>v$ and $u \leq_{i w} v$. Thus, denoting $u=x_{i_{1}} \ldots x_{i_{d}}$ with $i_{1} \leq \cdots \leq i_{d}$, there exist indices $1 \leq s<t \leq d$ such that

$$
\begin{aligned}
& i_{\alpha}=j_{\alpha} \\
& i_{s}<j_{s}, \\
& i_{\beta} \leq j_{\beta} \\
& i_{t}>j_{t},
\end{aligned} \quad \text { for all } 1 \leq \alpha \leq s-1,
$$

as in (2), (3) in the proof of Theorem 4.3. This implies

$$
2 \leq j_{s} \leq j_{t} \leq n-1
$$

since $1 \leq i_{s}<j_{s} \leq j_{t}<i_{t} \leq n$ by (7). Hence, the monomial $w=x_{j_{s}} x_{j_{t}}$ is a factor of $v$ of degree 2 in the variables $x_{2}, \ldots, x_{n-1}$.

Conversely, assume that $v$ has a factor $w$ of degree 2 in $x_{2}, \ldots, x_{n-1}$. Then there is a decomposition

$$
v=v_{1} x_{h_{1}} x_{h_{2}} v_{2}
$$

with $2 \leq h_{1} \leq h_{2} \leq n-1$ and with $v_{1}, v_{2}$ possibly trivial monomials ${ }^{\ddagger}$ satisfying $\max \left(v_{1}\right) \leq h_{1} \leq h_{2} \leq \min \left(v_{2}\right)$. We now set

$$
u=v_{1} x_{h_{1}-1} x_{n}^{b}
$$

where $b=d-\operatorname{deg}\left(v_{1}\right)-1$, so that $\operatorname{deg}(u)=d$. Note that $u>v$. Let us now start the computation of $\left(u^{\prime}, v^{\prime}\right)=\operatorname{sort}(u, v)$. On the level of index multisets, we have

$$
\left(\begin{array}{l}
U^{\prime} \\
V^{\prime}
\end{array}\right)=\left(\begin{array}{cccc}
V_{1} & h_{1}-1 & h_{2} & \ldots \\
V_{1} & h_{1} & \ldots & \ldots
\end{array}\right) .
$$

${ }^{\ddagger}$ Recall our conventions $\max (1)=0$ and $\min (1)=\infty$. 
Since $h_{2}<n$, it follows that $u^{\prime}>u$, whence also $v>v^{\prime}$ by Lemma 2.2. Summarizing, we found that $L(v)$ contains $u, v$ but not $v^{\prime}$. Therefore $L(v)$ is not sortable, as stated.

Corollary 5.3. Let $v \in S_{n, d}$. Then $L(v)$ is sortable if and only if $v=x_{1}^{a} x_{j} x_{n}^{b}$ for some $a, b \in \mathbb{N}$ and some index $j$ such that $1 \leq j \leq n$.

Compare with Proposition 3.2 (i) of [6], a closely related statement.

\section{The greatest common prefix}

In order to extend our sortability criterion to arbitrary lex-intervals of monomials, we shall need the notion of greatest common prefix of two or more monomials. Analogous notions appear in various contexts such as computer science, combinatorics on words, computational molecular biology and braid theory.

Definition 6.1. Let $u \in S$ be a monomial in $x_{1}, \ldots, x_{n}$. A prefix of $u$ is any factor $w$ of $u$ satisfying

$$
\max (w) \leq \min (u / w)
$$

Equivalently, if $u=x_{i_{1}} \cdots x_{i_{d}}$ with $i_{1} \leq \cdots \leq i_{d}$, a prefix of $u$ is a factor $w$ of the form

$$
w=x_{i_{1}} \cdots x_{i_{k}}
$$

for some $0 \leq k \leq d$.

Note that for each $0 \leq k \leq \operatorname{deg}(u)$, there is a unique prefix $w$ of $u$ of degree $k$. We now consider the case of two monomials; the extension to more monomials is straightforward.

Definition 6.2. Let $u, v \in S$ be monomials in $x_{1}, \ldots, x_{n}$. The greatest common prefix of $u, v$, denoted

$$
\operatorname{gcp}(u, v) \text {, }
$$

is the common prefix of $u, v$ of highest degree.

Note that $\operatorname{gcp}(u, v)$ divides $\operatorname{gcd}(u, v)$, the usual greatest common divisor of $u, v$. For example, if $u=x_{1} x_{2}^{2} x_{3}$ and $v=x_{1} x_{2} x_{3}^{2}$, then

$$
\operatorname{gcp}(u, v)=x_{1} x_{2}, \quad \operatorname{gcd}(u, v)=x_{1} x_{2} x_{3} .
$$

Here is an equivalent characterization of $\operatorname{gcp}(u, v)$. 
Remark 6.3. Let $u, v$ be monomials in $x_{1}, \ldots, x_{n}$. Then $\operatorname{gcp}(u, v)$ is the common factor $w$ of $u, v$ of highest degree satisfying

$$
\max (w) \leq \min (u / w), \quad \max (w) \leq \min (v / w) .
$$

Finally, let us observe that the notion of gcp allows a useful equivalent formulation of the lexicographical order.

Proposition 6.4. Let $v_{1} \neq v_{2} \in S_{n, d}$. Let $v_{0}=\operatorname{gcp}\left(v_{1}, v_{2}\right)$. Then $v_{1}>_{\text {lex }} v_{2}$ if and only $\min \left(v_{1} / v_{0}\right)<\min \left(v_{2} / v_{0}\right)$.

\section{The case of lex-intervals}

Given $v_{1} \geq v_{2}$ in $S_{n, d}$, we denote by $L\left(v_{1}, v_{2}\right)$ the lex-interval determined by $v_{1}, v_{2}$ with respect to the lexicographical order, namely

$$
L\left(v_{1}, v_{2}\right)=\left\{u \in S_{n, d} \mid v_{1} \geq u \geq v_{2}\right\} .
$$

Of course, lex-segments are lex-intervals: if $v \in S_{n, d}$, then

$$
L(v)=L\left(x_{1}^{d}, v\right) .
$$

In this section, we generalize Theorem 5.2 and determine which lex-intervals in $S_{n, d}$ are not sortable. Even though Theorem 5.2 will follow as an immediate corollary, we have treated it separately with an independent and simpler proof.

The case of arbitrary lex-intervals requires the notion of greatest common prefix introduced in the preceding section. Note $L(v, v)=\{v\}$ is sortable, since $\operatorname{sort}(v, v)=(v, v)$. Thus, we only need to examine the case where $v_{1}>v_{2}$.

Theorem 7.1. Let $v_{1}>v_{2}$ be monomials in $x_{1}, \ldots, x_{n}$ of degree $d$. Let $v_{0}=\operatorname{gcp}\left(v_{1}, v_{2}\right)$. Then $L\left(v_{1}, v_{2}\right)$ is not sortable if and only if the monomial $v_{2} / v_{0}$ has a factor $w$ of degree 2 such that $\max (w)<n$.

Proof. - Assume first that $v_{2} / v_{0}$ has a factor $w=x_{h_{1}} x_{h_{2}}$ with $h_{1} \leq h_{2}<n$. Since $v_{1}>v_{2}$, there are decompositions

$$
v_{1}=v_{0} w_{1}, \quad v_{2}=v_{0} w_{2}
$$

with $\max \left(v_{0}\right) \leq \min \left(w_{1}\right)<\min \left(w_{2}\right)$. Without loss of generality, we may assume that $w$ is the prefix of degree 2 in $w_{2}$. Thus, we may further decompose

$$
w_{2}=x_{h_{1}} x_{h_{2}} \overline{w_{2}}
$$


with $h_{1}=\min \left(w_{2}\right)$ and $\min \left(\overline{w_{2}}\right) \geq h_{2}$. We shall now find a special monomial $u$ in $L\left(v_{1}, v_{2}\right)$ such that $\operatorname{sort}\left(u, v_{2}\right)$ falls outside $L\left(v_{1}, v_{2}\right) \times L\left(v_{1}, v_{2}\right)$. Set

$$
u=v_{0} x_{h_{1}-1} x_{n}^{b}
$$

with $b=d-\operatorname{deg}\left(v_{0}\right)-1$, so that $\operatorname{deg}(u)=d$.

We first show, using Proposition 6.4, that $u$ belongs to $L\left(v_{1}, v_{2}\right)$. Indeed, on the one hand we have

$$
u>v_{2},
$$

since $v_{0}$ is a common prefix of $u, v_{2}$ and since $h_{1}-1=\min \left(w_{2}\right)-1$. On the other hand, in order to show

$$
v_{1} \geq u,
$$

we need to determine the greatest common prefix of $v_{1}, u$. First note that $\min \left(w_{1}\right) \leq h_{1}-1$, since $\min \left(w_{1}\right)<\min \left(w_{2}\right)=h_{1}$. We get:

$$
\operatorname{gcp}\left(v_{1}, u\right)= \begin{cases}v_{0} & \text { if } \min \left(w_{1}\right)<h_{1}-1, \\ v_{0} x_{h_{1}-1} & \text { if } \min \left(w_{1}\right)=h_{1}-1 \text { and } w_{1}>x_{h_{1}-1} x_{n}^{b}, \\ v_{0} x_{h_{1}-1} x_{n}^{b} & \text { if } \min \left(w_{1}\right)=h_{1}-1 \text { and } w_{1}=x_{h_{1}-1} x_{n}^{b}\end{cases}
$$

In either case, we easily conclude with Proposition 6.4 that $v_{1} \geq u$. Therefore $u \in L\left(v_{1}, v_{2}\right)$, as stated.

Let us now apply the sort operator to the pair $\left(u, v_{2}\right)$. On the level of index multisets, we have

$$
\left(\begin{array}{c}
U^{\prime} \\
V_{2}^{\prime}
\end{array}\right)=\left(\begin{array}{cccc}
V_{0} & h_{1}-1 & h_{2} & \cdots \\
V_{0} & h_{1} & \cdots & \cdots
\end{array}\right) .
$$

Since $h_{2}<n$, it follows that $u^{\prime}>u$, whence also $v_{2}>v_{2}^{\prime}$ by Lemma 2.2. Thus $L\left(v_{1}, v_{2}\right)$ contains $u, v_{2}$ but not $v_{2}^{\prime}$. It follows that $L\left(v_{1}, v_{2}\right)$ is not sortable, as stated.

- Conversely, assume that $v_{2} / v_{0}$ has no factor $w$ of degree 2 satisfying $\max (w)<n$. It follows that either $\operatorname{deg}\left(v_{2} / v_{0}\right)=1$, or else

$$
v_{2} / v_{0}=x_{j} x_{n}^{b}
$$

with $\max \left(v_{0}\right) \leq j \leq n$ and $b \geq 1$. We now show that, in each case, the interval $L\left(v_{1}, v_{2}\right)$ is sortable.

(1) Assume first $\operatorname{deg}\left(v_{2} / v_{0}\right)=1$. Then, since $v_{1}>v_{2}$, there are indices $\max \left(v_{0}\right) \leq h_{1}<h_{2} \leq n$ such that

$$
v_{1}=v_{0} x_{h_{1}}, \quad v_{2}=v_{0} x_{h_{2}} .
$$


It follows that $L\left(v_{1}, v_{2}\right)=v_{0}\left\{x_{h_{1}}, x_{h_{1}+1}, \ldots, x_{h_{2}}\right\}$. But then, it is clear that for any indices $i, j$ such that $h_{1} \leq i \leq j \leq h_{2}$, we have

$$
\operatorname{sort}\left(v_{0} x_{i}, v_{0} x_{j}\right)=\left(v_{0} x_{i}, v_{0} x_{j}\right) .
$$

Thus, $L\left(v_{1}, v_{2}\right)$ is sortable in this case.

(2) Assume now $v_{2} / v_{0}=x_{j} x_{n}^{b}$ with $\max \left(v_{0}\right) \leq j \leq n$ and $b \geq 1$. Since $v_{1}>v_{2}=v_{0} x_{j} x_{n}^{b}$, there is a decomposition

$$
v_{1}=v_{0} x_{h} \overline{v_{1}}
$$

with $\max \left(v_{0}\right) \leq h<j$ and $h \leq \min \left(\overline{v_{1}}\right)$. Note that the equality $h=j$ is excluded by our assumption $\operatorname{gcp}\left(v_{1}, v_{2}\right)=v_{0}$. With these values of $v_{1}, v_{2}$, we now describe all monomials in the lex-interval $L\left(v_{1}, v_{2}\right)$.

Claim. Let $u \in S_{n, d}$. Then we have

$$
v_{0} x_{h} \overline{v_{1}} \geq u \geq v_{0} x_{j} x_{n}^{b}
$$

if and only if $u=v_{0} x_{i} \bar{u}$ for some monomial $\bar{u}$ and some index $i$ such that $h \leq i \leq j$, and either

(i) $h=i$ and $\overline{v_{1}} \geq \bar{u}$, or else

(ii) $h<i \leq j$ and $\bar{u}$ is any monomial in $x_{i}, \ldots, x_{n}$ of appropriate degree.

Checking the claim is straightforward and left to the reader.

We now prove that $L\left(v_{1}, v_{2}\right)$ is sortable. So let $u_{1} \geq u_{2} \in L\left(v_{1}, v_{2}\right)$. Since $v_{1} \geq u_{1} \geq u_{2} \geq v_{2}$, there are decompositions

$$
u_{1}=v_{0} x_{i_{1}} \overline{u_{1}}, \quad u_{2}=v_{0} x_{i_{2}} \overline{u_{2}}
$$

such that $h \leq i_{1} \leq i_{2} \leq j$. Let us start the computation of $\left(u_{1}^{\prime}, u_{2}^{\prime}\right)=$ $\operatorname{sort}\left(u_{1}, u_{2}\right)$. On the level of index multisets, we have

$$
\left(\begin{array}{c}
U_{1}^{\prime} \\
U_{2}^{\prime}
\end{array}\right)=\left(\begin{array}{lll}
V_{0} & i_{1} & \cdots \\
V_{0} & i_{2} & \cdots
\end{array}\right)
$$

It follows that $u_{2}^{\prime}$ admits $v_{0} x_{i_{2}}$ as a prefix. Recalling the equality $v_{2}=v_{0} x_{j} x_{n}^{b}$, we now show that $u_{2}^{\prime} \geq v_{2}$.

(i) If $i_{2}<j$, then clearly $u_{2}^{\prime}>v_{2}$.

(ii) If $i_{2}=j$, then $u_{2}^{\prime} \geq v_{2}$ since $v_{2}$, by its specific structure, is the smallest monomial of its degree having $v_{0} x_{j}$ as a prefix. 
It follows from Lemma 2.2 that $v_{1} \geq u_{1}^{\prime} \geq u_{2}^{\prime} \geq v_{2}$, and hence that $u_{1}^{\prime}, u_{2}^{\prime}$ still belong to $L\left(v_{1}, v_{2}\right)$. Therefore $L\left(v_{1}, v_{2}\right)$ is sortable, as stated.

Corollary 7.2. The only sortable lex-intervals $L\left(v_{1}, v_{2}\right) \subset S_{n, d}$ with $v_{1}>v_{2}$ are those such that $\left(v_{1}, v_{2}\right)=\left(v_{0} w_{1}, v_{0} x_{j} x_{n}^{b}\right)$, where $b=d-\operatorname{deg}\left(v_{0}\right)-1$ and where $v_{0}, w_{1}$ are monomials satisfying

$$
\max \left(v_{0}\right) \leq \min \left(w_{1}\right)<j \leq n .
$$

\section{From lex to revlex}

Our aim here is to establish the analogue of Theorem 7.1 for intervals of monomials under the reverse lexicographical order on $S_{n, d}$. Instead of adapting our earlier proofs to this new setting, we shall develop tools allowing us to transfer knowledge between the lex and the revlex orders. The desired analogue will then directly follow from Theorem 7.1 using those tools.

Recall first the definition of the revlex order. Given $\mathbf{a}=\left(a_{1}, \ldots, a_{n}\right)$, $\mathbf{b}=\left(b_{1}, \ldots, b_{n}\right) \in \mathbb{N}^{n}$ such that $\sum_{i} a_{i}=\sum_{i} b_{i}=d$, we write

$$
x_{1}^{a_{1}} \cdots x_{n}^{a_{n}}>_{\text {rev }} x_{1}^{b_{1}} \cdots x_{n}^{b_{n}}
$$

if and only if the rightmost nonzero coordinate of $\mathbf{a}-\mathbf{b}$ is negative. Equivalently, let

$$
u=x_{i_{1}} \cdots x_{i_{d}}, v=x_{j_{1}} \cdots x_{j_{d}} \in S_{n, d}
$$

with $i_{1} \leq \cdots \leq i_{d}, j_{1} \leq \cdots \leq j_{d}$. Then $u>_{\text {rev }} v$ if and only if the rightmost nonzero coordinate of $\left(i_{1}-j_{1}, \ldots, i_{d}-j_{d}\right)$ is negative.

Informally, the lex order gives a premium in priority to $x_{1}$, then to $x_{2}$ and so on, whereas the revlex order puts a penalty in priority to $x_{n}$, then to $x_{n-1}$ and so on. In $S_{2,2}$ for instance, we have

$$
x_{1} x_{3}>_{\text {lex }} x_{2}^{2} \text { but } x_{1} x_{3}<_{\text {rev }} x_{2}^{2} .
$$

Note also that $x_{1}>\cdots>x_{n}$ for both orders.

\subsection{The automorphism $\sigma$}

A convenient way to compare the lex and revlex orders is through the $K$ algebra automorphism

$$
\begin{aligned}
\sigma: K\left[x_{1}, \ldots, x_{n}\right] & \longrightarrow K\left[x_{1}, \ldots, x_{n}\right] \\
x_{i} & \longmapsto x_{n+1-i} \forall i=1, \ldots, n .
\end{aligned}
$$


Indeed, for any monomials $u, v \in S_{n, d}$, we have

$$
u>_{\text {lex }} v \Longleftrightarrow \sigma(u)<_{\text {rev }} \sigma(v) \text {. }
$$

Since $\sigma^{-1}=\sigma$, this equivalence may as well be written in the form

$$
v>_{\text {rev }} u \Longleftrightarrow \sigma(u)<_{\text {lex }} \sigma(v) \text {. }
$$

\subsection{Revlex-intervals}

We now define intervals in $S_{n, d}$ under the revlex order.

Definition 8.1. Given $v_{1} \geq_{r e v} v_{2}$ in $S_{n, d}$, we denote by $R\left(v_{1}, v_{2}\right)$ the revlexinterval determined by $v_{1}, v_{2}$, namely

$$
R\left(v_{1}, v_{2}\right)=\left\{u \in S_{n, d} \mid v_{1} \geq_{r e v} u \geq_{r e v} v_{2}\right\}
$$

Lex-intervals and revlex-intervals may be compared as follows.

Lemma 8.2. For any $v_{1} \geq_{r e v} v_{2}$ in $S_{n, d}$, we have

$$
\sigma\left(R\left(v_{1}, v_{2}\right)\right)=L\left(\sigma\left(v_{2}\right), \sigma\left(v_{1}\right)\right)
$$

Proof. Let $u \in S_{n, d}$. Then $u \in R\left(v_{1}, v_{2}\right)$ if and only if $v_{1} \geq_{\text {rev }} u \geq_{\text {rev }} v_{2}$. Applying $\sigma$ and using (9) this in turn is equivalent to

$$
\sigma\left(v_{1}\right) \leq_{l e x} \sigma(u) \leq_{l e x} \sigma\left(v_{2}\right),
$$

i.e. to $\sigma(u) \in L\left(\sigma\left(v_{2}\right), \sigma\left(v_{1}\right)\right)$. It follows that $R\left(v_{1}, v_{2}\right)=\sigma^{-1}\left(L\left(\sigma\left(v_{2}\right), \sigma\left(v_{1}\right)\right)\right)$, whence the stated formula.

\subsection{Sort and $\sigma$}

Here we describe how sort and $\sigma$ interact with each other.

Lemma 8.3. For any $u, v \in S_{n, d}$, let $\left(u^{\prime}, v^{\prime}\right)=\operatorname{sort}(u, v)$. Then

$$
\operatorname{sort}(\sigma(u), \sigma(v))=\left(\sigma\left(v^{\prime}\right), \sigma\left(u^{\prime}\right)\right) .
$$

Proof. Denote $u v=x_{k_{1}} x_{k_{2}} \ldots x_{k_{2 d}}$ with nondecreasing indices $k_{i}$. Then by construction, we have

$$
\begin{aligned}
& u^{\prime}=x_{k_{1}} x_{k_{3}} \ldots x_{k_{2 d-1}} \\
& v^{\prime}=x_{k_{2}} x_{k_{4}} \ldots x_{k_{2 d}}
\end{aligned}
$$


Now $\sigma(u) \sigma(v)=\sigma(u v)=x_{n+1-k_{2 d}} x_{n+1-k_{2 d-1}} \ldots x_{n+1-k_{1}}$, here again with nondecreasing indices. Applying sort to the pair $(\sigma(u), \sigma(v))$, it follows that

$$
\begin{aligned}
& \sigma(u)^{\prime}=x_{n+1-k_{2 d}} x_{n+1-k_{2 d-2}} \ldots x_{n+1-k_{2}}=\sigma\left(v^{\prime}\right) \\
& \sigma(v)^{\prime}=x_{n+1-k_{2 d-1}} x_{n+1-k_{2 d-3}} \ldots x_{n+1-k_{1}}=\sigma\left(u^{\prime}\right),
\end{aligned}
$$

as stated.

A useful consequence is that $\sigma$ preserves the sortability property.

Proposition 8.4. Let $B$ be a subset of $S_{n, d}$. Then $B$ is sortable if and only if $\sigma(B)$ is sortable.

Proof. Since $\sigma^{-1}=\sigma$, it suffices to prove one direction. So assume that $B$ is sortable. Any element in $\sigma(B) \times \sigma(B)$ is of the form $(\sigma(u), \sigma(v))$ for some pair $(u, v) \in B \times B$. We claim that $\operatorname{sort}(\sigma(u), \sigma(v))$ still belongs to $\sigma(B) \times \sigma(B)$. Indeed, by Lemma 8.3, we have

$$
\operatorname{sort}(\sigma(u), \sigma(v))=\left(\sigma\left(v^{\prime}\right), \sigma\left(u^{\prime}\right)\right) .
$$

But $\left(u^{\prime}, v^{\prime}\right) \in B \times B$ since $B$ is sortable, whence $\left(\sigma\left(v^{\prime}\right), \sigma\left(u^{\prime}\right)\right) \in \sigma(B) \times \sigma(B)$. This shows that $\operatorname{sort}(\sigma(u), \sigma(v)) \in \sigma(B) \times \sigma(B)$, as claimed. Therefore $\sigma(B)$ is sortable.

\subsection{The greatest common suffix}

Definition 8.5. Let $u=x_{i_{1}} \cdots x_{i_{d}}$ be a monomial in $x_{1}, \ldots, x_{n}$ of degree $d$ with $i_{1} \leq \cdots \leq i_{d}$. Let $1 \leq k \leq d$. The $k$-suffix of $u$ is the degree $k$ monomial

$$
x_{i_{d+1-k}} \cdots x_{i_{d}}
$$

It may be characterized as the unique monomial $u_{0}$ of degree $k$ such that $u_{0}$ divides $u$ and $\min \left(u_{0}\right) \geq \max \left(u / u_{0}\right)$.

We now introduce the analogue for suffixes of the greatest common prefix.

Definition 8.6. Let $u, v \in S$ be monomials in $x_{1}, \ldots, x_{n}$. The greatest common suffix of $u, v$, denoted

$$
\operatorname{gcs}(u, v)
$$

is the common suffix of $u, v$ of highest degree.

Note that $\operatorname{gcs}(u, v)$ divides $\operatorname{gcd}(u, v)$, as was the case for $\operatorname{gcp}(u, v)$. Taking our earlier example, if $u=x_{1} x_{2}^{2} x_{3}$ and $v=x_{1} x_{2} x_{3}^{2}$, we have

$$
\operatorname{gcs}(u, v)=x_{3}
$$


Moreover, $\operatorname{gcs}(u, v)$ may be characterized as the common factor $w$ of $u, v$ of highest degree satisfying

$$
\min (w) \geq \max (u / w), \quad \min (w) \geq \max (v / w) .
$$

The following result shows that $\sigma$ transforms gcs into gcp.

Lemma 8.7. For all $v_{1}, v_{2} \in S_{n, d}$, we have $\sigma\left(\operatorname{gcs}\left(v_{1}, v_{2}\right)\right)=\operatorname{gcp}\left(\sigma\left(v_{2}\right), \sigma\left(v_{1}\right)\right)$.

Proof. This follows from the observation that, for any monomial $v$, a monomial $v_{0}$ is a suffix of $v$ if and only if $\sigma\left(v_{0}\right)$ is a prefix of $\sigma(v)$.

\subsection{Sortable revlex-intervals}

We are now ready to determine which revlex-intervals are sortable and which are not. The results will follow from Theorem 7.1, Corollary 7.2 and the above properties of the automorphism $\sigma$.

Theorem 8.8. Let $v_{1}>_{r e v} v_{2}$ be monomials in $x_{1}, \ldots, x_{n}$ of degree $d$. Let $v_{0}=\operatorname{gcs}\left(v_{1}, v_{2}\right)$. Then $R\left(v_{1}, v_{2}\right)$ is not sortable if and only if $v_{1} / v_{0}$ has a factor $w$ of degree 2 such that $\min (w)>1$.

Proof. By Proposition 8.4, the non-sortability of $R\left(v_{1}, v_{2}\right)$ is equivalent to that of $\sigma\left(R\left(v_{1}, v_{2}\right)\right)$. Now $\sigma\left(R\left(v_{1}, v_{2}\right)\right)=L\left(\sigma\left(v_{2}\right), \sigma\left(v_{1}\right)\right)$ by Lemma 8.2, and the non-sortability of this lex-interval may be determined using Theorem 7.1. By Lemma 8.7, we have $\operatorname{gcp}\left(\sigma\left(v_{2}\right), \sigma\left(v_{1}\right)\right)=\sigma\left(v_{0}\right)$, where $v_{0}=\operatorname{gcs}\left(v_{1}, v_{2}\right)$. By Theorem 7.1, $L\left(\sigma\left(v_{2}\right), \sigma\left(v_{1}\right)\right)$ is non-sortable if and only if $\sigma\left(v_{1}\right) / \sigma\left(v_{0}\right)$ has a factor $w^{\prime}$ of degree 2 such that $\max \left(w^{\prime}\right)<n$. Let $w=\sigma\left(w^{\prime}\right)$. Now

$$
\max \left(w^{\prime}\right)<n \Longleftrightarrow \min (w)>1 .
$$

Thus, applying $\sigma$, we have that $L\left(\sigma\left(v_{2}\right), \sigma\left(v_{1}\right)\right)$ is non-sortable if and only if $v_{1} / v_{0}$ has a factor $w$ of degree 2 such that $\min (w)>1$. It follows that $R\left(v_{1}, v_{2}\right)$ is not sortable if and only if the latter condition holds, as stated.

Corollary 8.9. The only sortable revlex-intervals $R\left(v_{1}, v_{2}\right) \subset S_{n, d}$ with $v_{1}>_{\text {rev }}$ $v_{2}$ are those such that $\left(v_{1}, v_{2}\right)=\left(x_{1}^{b} x_{j} v_{0}, w_{1} v_{0}\right)$, where $b=d-\operatorname{deg}\left(v_{0}\right)-1$ and where $v_{0}, w_{1}$ are monomials satisfying

$$
1 \leq j<\max \left(w_{1}\right) \leq \min \left(v_{0}\right) .
$$

\section{References}

[1] E. De Negri, Toric rings generated by special stable sets of monomials, Math. Nachr. 203 (1999) 31-45. 
[2] V. Ene, J. Herzog, Gröbner bases in commutative algebra. Graduate Studies in Mathematics, 130. American Mathematical Society, Providence, RI, 2011.

[3] J. Herzog, T. Hibi, Discrete Polymatroids, J. Algebraic Combinatorics 16 (2002) 239-268.

[4] M. La Barbiera, A note on unmixed ideals of Veronese bi-type ideal, Turk. J. Math. 37 (2013) 1-7.

[5] B. Sturmfels, Gröbner bases and convex polytopes. University Lecture Series, 8. American Mathematical Society, Providence, RI, 1996.

[6] V. Bonanzinga, Principal Borel ideals and Gotzmann ideals, Arch. Math. 81 (2003) 385-396.

Vittoria BONANZINGA,

Department DIIES,

Mediterranean University of Reggio Calabria,

via Graziella (Feo di Vito) 89100 Reggio Calabria, Italia.

Email: vittoria.bonanzinga@unirc.it

Shalom ELIAHOU,

Univ. Littoral Côte d'Opale, EA 2597

LMPA - Laboratoire de Mathématiques Pures et Appliquées Joseph Liouville,

F-62228 Calais, France

CNRS, FR 2956, France

Email: eliahou@lmpa.univ-littoral.fr 